\title{
林冠附生植物生态学研究进展
}

\author{
刘文耀 1 3 马文章 1 , 2 杨礼攀1 2 \\ （1 中国科学院西双版纳热带植物园昆明分部, 昆明 650223) (2 中国科学院研究生院 北京 100039)
}

(3 科廷理工大学 珀斯 WA6845, 澳大利亚)

摘 要 林冠附生植物及其枯死存留物是构成山地湿性森林生态系统中生物区系、结构和功能的重要组分。由于 在林冠攀爬技术上的限制，过去对林冠附生植物在生态系统结构和功能过程中的作用未能引起足够的重视。近 20 年来, 随着对林冠生物多样性及其在生态系统功能过程影响的认识和研究技术上的提高, 对林冠附生生物的研究 已逐步从个体水平转移到系统水平上。有关林冠附生植物多样性、生物量及其生态学效应已成为近年来国际上新 兴研究领域—“ 林冠学” 的研究热点之一。许多研究表明 林冠附生植物在生态系统水平上的交互作用比它们的 解剖、形态和生理特征更为重要。国外大量的研究结果表明 林冠是一个适合于许多生物种类生存的场所, 其数量 比想象的更为丰富。在全球范围内估计有 29500 余种附生植物, 其中维管束附生植物的种类高达 24000 种, 约占 总维管束植物种类的 10\%。林冠附生物的生物量在世界各地森林中存在较大的差异, 其范围在 $105 \sim 44000 \mathrm{~kg}$ $\mathrm{hm}^{-2}$ 之间 其中在一些热带和温带天然老龄林中林冠附生物的生物量超过了宿主林木的叶生物量。林冠附生植物 还具有较大的叶面积指数 ( $L A I)$ 。林冠附生物丰富的物种组成、较高的生物量、独特的生理形态特征以及它们分布 于森林与大气相互作用的关键界面，使得它们在生态系统物种多样性形成及其维持机制、养分和水分循环、指示环 境质量等方面具有重要的作用。林冠附生植物及其枯死残留物具有较强的能力吸收雨水和空气中的营养物质,在 林冠层中形成一个潮湿的环境促进氮固定 林冠附生植物群落还能为生存于林冠的其它生物 (如鸟类、哺乳动物、 两栖动物、爬行动物和昆虫等)提供食物和栖息场所。林冠附生植物的生长发育与分布格局除与宿主有关外，还受 到环境因素(气候、地形、微生境条件等)和人为干扰的影响。由于世界各地森林类型多样和环境条件各异,目前国 际上有关附生物的研究仍十分活跃 建立了林冠研究网络, 研究不同类型森林中附生植物及其枯死残留物的动态 及其与群落特征、环境因子的数量关系, 探讨、交流和发展有效的标准测量方法和技术是该领域研究的主要内容。 国内对林冠附生植物生态学的研究刚刚起步, 有待于今后加强该领域的研究。

关键词 林冠 附生植物 物种多样性 生物量 生态功能 环境因素

\section{ADVANCES IN ECOLOGICAL STUDIES ON EPIPHYTES IN FOREST CANOPIES}

\author{
LIU Wen-Yao ${ }^{1,3}$ MA Wen-Zhang ${ }^{1,2}$ and YANG Li-Pan ${ }^{1,2}$ \\ (1 Xishuangbanna Tropical Botanical Garden , Chinese Academy of Sciences , Kunming 650223 , China) \\ (2 Graduate School of Chinese Academy of Sciences , Beijing 100039 , China) \\ (3 Curtin University of Technology, Pereh WA6845, Australia)
}

\begin{abstract}
Canopy-dwelling epiphytes and their associated dead organic matter are important floristic, structural and functional components in montane moist forests. Because of the difficulties related to access , the biodiversity and ecosystem-level functional attributes of epiphytes have received little attention in forests. With an increase in our understanding of epiphytic biodiversity and their roles in ecosystem-level interactions , combined with improved access to the forest canopy, studies on epiphytic organisms in forest canopies have progressed from the individual level to the ecosystem level. Biodiversity and biomass of epiphytes and their functional roles at the ecosystem-level are becoming a hot topic of recent study. Recent work in tree canopies in a variety of forest types, however, has pointed out that the role of epiphytes in ecosystem-level interactions are more important than previously thought due to their anatomical , morphological , and physiological characteristics. Considerable research conducted worldwide has shown that the forest canopy is a favorable habitat supporting a much richer epiphytic flora than previously thought. It was estimated that there are 29500 epiphytic species , including 24000 vascular epiphytes that account for $10 \%$ of the total vascular species in the world. There are large differences in the epiphytic biomass of forests worldwide ranging from $105-44000 \mathrm{~kg} \mathrm{hm}^{-2}$. The biomass of
\end{abstract}


epiphytic material of forest canopies was greater than the leaf biomass of host trees in some old growth forests . Epiphytes have a tremendous leaf area index $(L A I)$. Epiphytes play an important role in biodiversity, water and nutrient cycling in forest ecosystems, and are sensitive indicators of environment change, due to rich species, high biomass and special morphological traits and their position at the forest-atmosphere interface. The epiphyte community also provides a source of food and habitat for a variety of birds , mammals , amphibians, reptiles, and insects.

Species composition, growth and distribution of epiphytes are influenced by environmental factors and human disturbance. Research on epiphytes in forest canopies still is very active due to a lack of field data at the ecosystem level for a lot of forest types under different environmental conditions in many regions. International forest canopy networks have been established worldwide. To understand the relationship among community characteristics, environmental factors and epiphyte dynamics , and develop effective and standard methods and technologies for the study of forest canopies , more work is needed in this field. In China , there has been very little work on the ecology of epiphytes in forest canopies, and this remains a challenging and fruitful area for future research.

Key words Forest canopy , Epiphytes , Species diversity , Biomass , Ecological functions , Environmental factors

林冠是森林-大气相互作用的关键生态界面，在 生态系统生物多样性形成与维系，物质、能量交换过 程中发挥着重要的作用。然而,由于过去人们在认 识上、技术上和其它方面原因的限制，对林冠层的组 成、空间格局和生态学过程了解很少。近 20 年来, 随着全球变化、生物多样性和生态系统生态学研究 等全球性重大项目的开展, 以及多学科研究技术的 发展, 目前学术界已经将生物多样性的概念从地表/ 地下延伸到森林的林冠层。目前，国际上对林冠的 概念，已从过去简单的森林顶层 (Roof of the forest) (Parker, 1995) 发展为包括森林中的所有叶片、枝 条、小枝以及各种附生的有机体及其枯死残留物的 总和,也即森林中地表以上的所有植冠的集合 (Parker , 1995 ; Basset et al.,2003b ; Ozanne et al. , 2003)。有关林冠生态学的研究已经在欧、美、亚洲 的热带和温带地区不同类型的森林中开展, 产出了 一些较为重要的研究成果,包括在 Science 等重要刊 物上发表的林冠生态学研究论文数量也在与日俱增 (Nadkarni ,1981；Ozanne et al.,2003)；一些致力于 林冠生态学研究的国际组织(如国际林冠网络 (International Canopy Network，ICAN)、国际林冠塔吊网 络 (International Canopy Crane Network, ICCN)、全球林 冠项目 (Global Canopy Program, GCP) 也应运而生 (Basset et al ，2003c)。2005 年在 Nature 和 Science 两大世界著名的刊物上分别以焦点新闻的方式对正 在进行的有关林冠生物多样性、林冠与全球气候变 化等研究项目进行了报道 (Hopkin，2005; Pennisi， $2005 ）$, 说明林冠学研究已经成为目前国际上生物多 样性、全球变化研究中的热点问题。此外,从 1997
年以来, 国际林冠学 (Forest canopy) 大会每两年举行 一次, 2005 年在德国莱比锡大学召开了第四届, 2007 年将在印度班加洛尔召开第五届国际林冠学大会。

以各种附生生物 (尤其是附生植物) 及其枯死残 留物为主组成的附生物质, 是热带、亚热带和温带山 地森林林冠中重要的组成部分，构成了明显的景观 结构特征(Nadkarni ,1984; Lowman \& Nadkarni ,1995)。 这些附生物质 (Epiphytic material, EM) 的存在, 不仅 使山地森林生态系统具有很高的生物多样性和生物 量, 而且影响着生态系统的养分与水分平衡、生物地 球化学循环以及生产力 (Pócs ,1980；Brown ,1982 ;Vitousek \& Sanford , 1986 ;Veneklaas , 1991 ;Lowman \& Ndkarni ,1995)。从 20 世纪 70 年代以来, 在南美、非 洲和北欧等一些国家和地区对热带、温带森林中附 生物及其生态学效应进行了研究,1995 年由 Lowman 和 Nadikarni 编著出版的 Forest Canopy 一书, 标志着 国际上对林冠附生物和它们在山地森林生态系统中 的作用作为一个重要的研究内容而在世界各地广泛 开展。目前, 国外在世界近 70 个地区正在进行着有 关附生物的长期研究和探索 (Lowman, 2001)。本文 对近年来林冠附生植物组成、结构及其生态学效应 的研究进展作一系统的综述。

\section{1 林冠附生物的定义及研究简史}

附生生物是一类生活于植物活体或其残体上 的、其生命活动不从宿主载体上吸取营养与水分的 特殊有机生命体或生物（Barkman，1958），半附生生 物生命周期中的空中阶段 (Aerial phases) 也包含在 这个定义中 (Wolf，1993a）。随着生物多样性、系统 
生态学和全球变化等全球性重大研究项目的开展, 人们发现除了林冠附生生物外, 存留在林冠中枯死 的附生生物残体、分解或半分解的腐殖质也具有重 要的生态功能。因此, 国际上提出了林冠附生物质 的概念，目前国际上普遍认同的林冠附生物质或附 生物是指由依附在树木、岩石表面的,由活着、死亡 的维管束和非维管束植物，以及与之相对应的碎屑、 微生物、无脊椎动物、真菌和林冠腐殖质等组成的混 合物 (Jenik, 1973)。附生植物及其枯死物是构成林 冠附生物的主要成分。

自 19 世纪以来，热带雨林中丰富的附生植物种 类与个体数量以及多样化的生长型 (Growth forms) 吸 引了一些植物学家的注意。但是, 由于林冠攀爬技 术的限制和认识方面的原因, 早期的研究主要局限 于对林冠大量的附生植物现象的描述。到了 20 世 纪 70 年代,Perry (1978) 创造了单绳攀爬技术 (Single rope technique) ,使得研究者能够进入到林冠层中开 展附生生物的调查研究。随后的相关报道大致以 10 年左右的间隔出现。20世纪 70 年代左右, 研究 集中在林冠底层附生植物的区系、盖度和生物量这 3 个方面。位于美国佛罗里达的 Marie Selby 植物园 从 1975 年开始收集附生植物标本, 至今总共采集到 29000 多种兰科植物和 8000 多种凤梨科植物 (Lowman，2001)。在 1975 年,美国创办了重点收集有关 附生植物研究的期刊 Selbyana, 其中关于附生植物 群落的研究主要集中在对附生植物种群建立、生长 和演替机制的研究, 并探讨其在森林生态系统中所 演绎的功能角色。20世纪 70 年代末, 单绳攀爬技 术的应用使得有关附生植物的研究逐渐成为学术界 上的一个热点 (Perry, 1978), 可以说这一技术的应用 成为附生植物研究史上的一次飞跃, 随后各国学者 广泛地在世界各地开展研究, 他们在热带地区建立 了 70 多个实验点对附生植物的物种多样性、生物量 及其生态效应等方面进行长期的研究观察 (Lowman，2001）。在过去的 20 年中,随着林冠研究者数 量的增加以及林冠接近技术的升级, 有关林冠研究 的文章增加了近 10 倍 (Lowman ,2001)。

然而，相对于陆生植物来说，林冠附生植物仍显 得很难接近, 其群落的组成成分很少为人们系统地 研究。根据有关学者 (Barker \& Pinard ,2001) 的统 计:在附生植物的研究史上, 从林地表面使用修枝 剪、望远镜和摄影等地面接近技术 (Ground access technique) 完成森林附生物的调查和采样工作的科 学工作者约占总数的 $27.5 \%$, 紧随其后的是单绳攀
爬技术(约占 $19.5 \%$ )。如何快捷有效地接近林冠 层一直困扰着各地的研究者。近年来, 林冠科学工 作者将起重吊塔 (Crane)、空中走廊 (Tower/Walkway) 和遥感 (Remote sensing) 等多种手段应用于林 冠生态学领域 (Barker \& Pinard，2001) ,才使得森林 附生物在森林生态系统中对物种多样性维系的作用 (Lowman , 2001 ; Nadkarni et al . ,2001)、对生物圈养 分和水分循环的贡献 (Jarvis，2000) 和自身强大的 生物指示功能（Lugo \& Scatena，1992；Sloof，1993； Loppi et al. , 1998 ; Wolf et al. 2003 ; Kreft et al. , 2004) 逐渐为保护生物学、生态学和水文学等领域 所重视。

尽管目前有关森林附生物的研究成为热点，然 而在研究的过程中也遇到一些制约调查工作大规模 开展的难题，如宿主树木枝干的复杂结构、采样方法 的标准化 (Nieder \& Zotz，1998) 等问题, 尚有待于在 研究过程中不断地探索。

\section{2 林冠附生植物的物种多样性}

林冠是陆地森林生态系统物种最丰富、同时也 是最濒危的生境之一，对维系生态系统生物多样性 具有重要作用。据估计 地球上大约 $40 \%$ 的生物种 类出现在林冠中 (Hammond et al . , 1997 ; Rodgers \& Kitching , 1998) 其中，大约有 $10 \%$ 的物种只分布于 林冠层(Hammond et al. , 1997)。Ozanne 等(2003)认 为，在维管束植物中有 $10 \%$ 的种类附生于林冠层 中。Kress (1986) 曾估计在全球范围内，维管束附生 植物的种类为 24000 种, 而 Gentry 和 Dodson (1987) 在把半附生植物也纳入统计范围之后, 估计在全球 范围内共存有来自于 83 个科的 29500 余种附生植 物。在热带地区，附生植物占据了维管束植物种类 的 25\% (Nieder et al ，2001)。Ibisch (1996) 在玻利 维亚的 Sehuencas 和 Carrasco 两个位于山地雨林中的 国家公园，根据调查结果得到的种-面积曲线，推测 出在 $1 \mathrm{hm}^{2}$ 的面积上存在着 1000 余种附生植物, 这 些附生植物在维系该地区植物种类多样性方面起着 重要的作用。Nadkarni (2001) 等对巴拿马、秘鲁、厄 瓜多尔、哥斯达黎加和委内瑞拉等地的山地雨林研 究后表明 林冠附生维管束植物的种类数量可以占 到当地维管束植物物种数的 $12 \% \sim 50 \%$ 。在厄瓜 多尔的热带雨林中，附生植物的物种数量在局部区 域内可以超过其它生命形式的物种（Kreft et al. , 2004)。此外 林冠丰富的附生物为其它生物的生 存、繁衍提供了良好的场所,研究表明全球有 $20 \%$ 
$\sim 25 \%$ 的无脊椎动物仅出现在林冠中 (Sorensen, 2003）超过 25\%的种类为植食者 (Herbivores) (Basset et al . ,2003a) 其中相当一部分为植物的授粉者。

近 10 年来在附生植物集中分布的热带、亚热带 地区关于附生植物种类的研究报道很多。在已有的 多样性报道中, 存在着两种采样单位的研究数据, 一 种是以单位面积的实验样地作为统计数据的单位, 比如 Kreft 等(2004) 在厄瓜多尔 Tiputini 生物多样性 研究站面积仅为 $0.1 \mathrm{hm}^{2}$ 的实验样地上, 记录了分 别属于 146 种、共 8762 株附生植物个体, 而在该研 究站共计 $650 \mathrm{hm}^{2}$ 面积上的调查结果表明, 该区域 内附生植物物种总数达 313 种; Nieder 等在 Sehuencas 面积为 $0.1 \mathrm{hm}^{2}$ 的样地上竟发现了 230 种附生维 管束植物 (Nadkarni，2001)。Annasel 和 Parthasarathy (2001) 在印度 Ghats 山脉地区 $30 \mathrm{hm}^{2}$ 的实验样地中 13445 棵胸径大于 $30 \mathrm{~cm}$ 的 601 棵宿主树和 3487 棵 宿主藤本上发现了分属 19 个属和 10 个科的 26 种 附生植物。另一种则是以特定的附生植物宿主树为 统计单位进行调查, Pentecost (1998) 在乌干达的 Rwenzori 山区对 Phillipia 群落中的隐花附生植物进 行调查时, 在 1 棵附生植物最多的宿主树上发现了 29 种不同的隐花附生植物，尽管作者认为这与 Wolf (1993b) 在安第斯山 4 棵树上发现的 150 种附生植 物相比显得少, 他将这种原因归结于树皮酸碱度对 附生植物多样性的制约，并认为是酸性树皮导致隐 花附生植物多样性的减少; Höfstede 等 (2001) 在新 西兰温带雨林的 3 棵大树 (1 棵 Nothofagus menziesii， 2 棵 Dacrycarpus dacrydioides) 上发现了 61 种维管束、 94 种非维管束附生植物, 显示出当地惊人的附生植 物物种多样性。

与原生林相比, 受干扰后形成的次生林中附生 植物在物种多样性上存在着巨大的差异。Hall （1978）曾报道过在加纳的一处次生林仅有 109 种维 管束附生植物, 而在附近的原生林中却有 504 种, 附 生植物的多度在次生林中较原生林少了 4 倍。 Barthlott 等 (2001) 对委内瑞拉 La Carbonera 地区附 生植物的多样性调查后发现: 原生林中有 178 种附 生植物, 而次生林中仅有 81 种, 且它们各自在组成 上存在巨大的差异。表现出后者多分布于凤梨科植 物, 而蒝类和兰科植物较少。Wolf (2005) 通过对墨 西哥 Chiapas 高地松栎林 (Pine-Oak forest) 的调查显 示, 随着人为干扰强度的增加 林地栎类树木密度和 大径级树木的减少，附生植物物种数明显的降低, 而 且, 喜湿的附生植物逐渐消失。

\section{3 林冠附生物的生物量及其结构}

林冠附生植物不仅具有丰富的多样性，而且以 附生植物及其枯死残留物为主构成的林冠附生物还 具有很高的生物量 (Höfstede et al ， 1993 ; Peck \& McCune，1998）。目前有关定量分析估计林冠附生 物生物量的方法主要是主观目测或非随机地从树上 采取枝条或对大径级宿主树的全树收获（Nadkarni et al. ，2004），通常都采取把采样树分成树干、树枝 等几个部分，再分别进行测量（Wolf，1993a，1993b； Økland，1994; Hölscher，2004）;也有通过树枝的分 枝等级情况而定的，如 Annasel 和 Parthasarathy (2001) 将每棵树划分成 4 个区域:主干区、第一、第 二和第三分枝，再对每级分枝上的附生植物进行单 独的测定, 其研究结果显示: 绝大多数 $(65 \%)$ 的附 生植物分布在主干上，第一、第二和第三分枝上分别 占 $17.4 \% 、 8.3 \%$ 和 $7 \%$ 。

从近年来报道的文献看，林冠附生物的生物量 在世界各地森林中存在较大的差异。从喀麦隆南部 Bakundu 森林中附生苔藓与附生蕨类 $105 \mathrm{~kg} \mathrm{hm}$ 年生 物量(Songwe et al., 1988)到哥伦比亚的安第斯山 $44000 \mathrm{~kg} \mathrm{hm}^{-2}$ 不等 (Höfstede et al. , 1993)。早在 1975 年 Golley 在巴拿马有关附生物生物量 $1400 \mathrm{~kg}$ $\mathrm{hm}^{-2}$ 的纪录,Edward 和 Grubb于 1977 年在新几内亚 山地森林所获得的数值是 $3400 \mathrm{~kg} \mathrm{hm}^{-2}$ (Edward \& Grubb , 1977)。Pócs (1980) 在坦桑尼亚的苔藓森林 (Mossy forest) 中测出 $14000 \mathrm{~kg} \mathrm{hm}^{-2}$ 的数据 ( Nadkarni , 1984)。Tanner (1980) 在牙买加的山地森林 中观测到的生物量为 $4 \sim 180 \mathrm{~kg} \mathrm{hm}^{-2}$, 约占当地地 上植被总生物量的 0 3\% ; Venklaas (1991) 在哥伦 比亚低地雨林中测定的维管束和非维管束附生植物 的生物量每年为 $220 \mathrm{~kg} \mathrm{hm}^{-2}$ 约占地上植被总生物 量的 3\%。Hölscher 等 (2004) 在哥斯达黎加的山地 森林中测出维管束和非维管束附生植物 (苔藓、地 衣、䓲类和附生凤梨科植物) 的生物量高达 $1920 \mathrm{~kg}$ $\mathrm{hm}^{-2}$ 。Höfstede 等 (2001) 在新西兰的温带雨林中，把 所获取的单位面积上的附生物质生物量推算到整棵 树上。在所测定的 3 棵树上得到每棵树上高达 400 $\mathrm{kg}$ 的生物量, 这一数值远远大于了 Nadkarni (1984) 所估测的 $114 \mathrm{~kg}$ 。这些巨大的差异一方面来自于所 处地理环境的不同，同时也来自于研究的附生物类 别的不同。其次，同一地区原生林与次生林之间 林 冠附生物生物量也存在较大的差异。Nadkarni 等 (2004) 在哥斯达黎加的研究发现附生物在原生林中 
的生物量是次生林中的 $40 \sim 100$ 倍, 其中在原生林 中的林冠腐殖质、残留的凋落物等占了林冠附生物 总生物量的 $60 \%$, 而在次生林中这部分林冠枯死有 机物所占的比例很小。在墨西哥 Chiapas 高地松栎 老龄林中, 附生植物生物量达 $3218 \mathrm{~kg} \mathrm{hm}^{-2}$, 而在 本地区遭受人为干扰影响后, 附生植物生物量随着 受干扰的程度而不同,最少的只有 $7.8 \mathrm{~kg} \mathrm{hm}$-2 (Wolf , 2005)。

\section{4 林冠附生植物群落的生态功能}

林冠附生物之所以一直吸引着人们的研究目 光，一个很重要的原因就是在于附生植物及其枯死 残留物具备多方面的生态功能，对森林生态系统生 物多样性形成及其维持、养分和水分循环、以及指示 环境质量等方面具有重要的作用 然而，人们对其中 的部分生态功能缺乏了解。根据近年来国际上有关 林冠附生物的研究, 它的生态功能主要表现在森林 生态系统水分、养分循环以及对环境变化的指示作 用等(Lowman \& Nadkarni，1995; Lowman，2001)。

首先，附生物群落的存在对于森林生态系统的 水分循环起到了重要的作用, 这主要是由其生理特 性决定的。Nadkarni (1984) 曾通过实验测定出附生 苔藓能够储存达自身干重 2 5 倍的水分, 关于这一 数值也有高达 13 倍的报道 (Silvola \& Aaltonen, 1984)。附生苔藓植物在密集分布时, 其吸水量可达 植物体自身干重的 $15 \sim 20$ 倍 (Silvola \& Aaltonen, 1984 ; 刘文耀 ,2000)。Barthlott 等 (2001) 认为附生物 群落的存在可为森林生态系统在干旱季节中提供水 分。在同一树冠内，附生物可使周围环境的水分损 失较没有附生物群落存在时平均降低 20\%（Stuntz et al ，，2002）。Weathers (1999) 曾用 电容” 来形容 林冠附生物对季节降水的调控作用，它们的存在可 以控制雨季带来的洪水和侵蚀, 并为旱季储存水分。 Hölscher 等 (2004) 认为附生物相对较大的咜水能力 (自身干重的 $400 \%$ ) 并不能完全发挥在与之相对应 的降雨截留能力上, 因为他们的实验数据显示在哥 斯达黎加山地雨林中的附生苔藓群落在当地总降雨 截留中仅仅起到了 $6 \%$ 的作用。这一点从侧面说明 了林冠附生物对云雾水表现出更佳的截留容载作 用。尽管云雾水仅占进入森林生态系统降水量的 $5 \%$ 20\% , 但森林中大多数植物的生长都依赖着这 部分水分，原因在于这些水分中所蕴含的铵离子和 硝酸根离子的浓度是其它形式水分的好几倍 (Benzing , 1998)。林冠附生物对这些云雾水的吸收能使
森林在降雨偏少的旱季不至受到水分胁迫的影响， 并保持一定的空气湿度, 缓和水分因子对森林生态 系统的限制。

其次，在养分循环方面，由于林冠附生植物没有 与地面相连接的根系, 附生植物对大气悬浮物的吸 收是无机养分的物质来源。它们在干季附生物质完 成对营养元素的咜存, 而在雨季又通过雨水的淋溶 使这部分养分汇入森林生态系统的养分循环中。除 此之外, 活附生植物及其枯死残留物所留下的附生 物垫层还通过宿主树木根系的吸收和凋落这两种途 径进入陆生植物的养分循环 (Nadkarni \& Matelson , 1992)。在通常情况下 林冠附生物所具备的这部分 养分仅占森林生态系统中总生物量中养分含量的 2\%（Nadkarni，1984），但这部分养分相对于为巨大 的树干木质部所占据着的养分而言, 能够迅速地被 淋溶和分解，从而对森林生态系统养分循环有更大 的意义。根据 Coxson (1991) 的测量数据, 在热带山 地雨林中林冠附生植物群落的叶面积指数 ( $L A I)$ 高达 160 , 反映出附生植物具有特别大的表面积, 为 它们吸收大气悬浮物中的养分元素提供了有利的条 件。而有关定量分析宿主树木凋落物的研究 (Hietz et al . 1999) 和使用同位素将大气中氮元素同陆地 土壤氮元素区分开的实验 (Field \& Dawson , 1998) 都证明了附生植物具有直接吸收大气中的水分和养 分元素的功能。因为附生植物中苔藓层的毛管作用 能够有效地减少土壤水分中矿物养分的流失, 进一 步增加营养物质在附生植物组织中的保留 (汪庆 等，1999），这种功能不容低估，在很多热带潮湿森 林中，附着在树干树枝上的活附生植物和死亡的有 机物质构成地上生物量和养分库的重要部分, 占到 叶中总养分含量的 45\%（Nadkarni，1984）。通过对 北方温带雨林的研究表明，虽然林冠附生物的生物 量仅占森林地上部分生物量的 $2 \%$,但它们对森林 生态系统营养库的贡献却是宿主叶片生物量的 4 倍 (Nadkarni ,1985)。

不仅如此，附生植物还为森林中的陆生植物提 供养分,Nadkarni（1981）发现有众多树种在极潮湿 的环境下能够生长出林冠根 (Canopy roots) 以吸收由 附生植物所产生的林冠腐殖质中的养分, 而不仅仅 是为了截获树木上的调落物。在哥斯达黎加的山地 森林中一棵树上的直径小于 $2 \mathrm{~mm}$ 林冠根的生物量 超过了根部周围的土壤重量, 说明附生植物比地表 土壤更能为陆生植物提供养分 (Coxson \& Nadkarni， 1995)。最近的研究发现，一些非维管束附生植物能 
够在干旱之后的湿润条件下释放大量的有机物质如 单糖和多元醇 (Mitchell et al. ,2005) , 有人推测附生 植物合成的这些高浓度化合物一方面是为了维持细 胞质的稳定，同时更重要的是这些所谓的代谢物的 数量每年可达 $122 \mathrm{~kg} \mathrm{hm}^{-2}$, 这对林冠养分循环和生 态系统养分供给能力起到了重要的作用 (Coxson , 1991)。

其三，林冠附生植物对环境变化和人为干扰比 较敏感, 具有指示环境质量的功能。由于附生植物 群落的叶表面积很大, 没有发达的根系, 其养分与水 分的吸收均由叶面完成, 其生长环境局限于与外界 无机环境的交界处, 直接暴露在不断变化的环境中， 这些在生理形态特征使得附生植物比其它形式的生 物对外界环境的变化更敏感 (Kreft et al ，2004) ,对 气候变化响应也特别明显 (Nadkarni \& Solano, 2002），这使得它们具备指示环境质量状况的潜在特 性，加之由于林冠附生植物种类繁多，各式各样的生 活型使得它们能够有效地占据不同类型的生境 （Nadkarni \& Matelson，1989）。正是因为在这种生态 位细化的前提下，一旦环境发生改变 就必然会影响 部分优势种组成的改变, 通过观察对比分析这种优 势附生植物在时间和空间尺度上的改变, 就能在一 定程度上归纳总结出环境质量的变化, 从而发挥其 环境指示剂的功能。

Benzing (1998) 认为由于林冠附生植物对水分 因子极其敏感的特性, 全球气候变化所带来的降水 格局的变化将直接导致附生植物分布的变化, 甚至 使部分种群消失。以苔藓为代表的附生植物有着很 多作为环境质量变化的生物指示剂的独特优点: 首 先它们分布广泛, 不受取样范围局限, 且它们的形态 不随季节变化, 所以终年都能够有效地吸收大气中 的养分元素; 其次它们不具备表皮结构, 养分元素粒 子能够轻易地渗透细胞壁, 在植物体中积聚 (Sloof, 1993 ; Berg \& Steinnes , 1997 ; Szczepaniak \& Biziuk , 2003 ）除此之外它们还具有相当长的寿命, 发挥指 示作用的时间较长。相对于陆生的苔藓植物而言, 附生苔藓植物因不受到土壤养分的干扰而更能较好 地监测环境中的金属离子与元素的变化, 起到生物 指示剂的作用。随着人类对环境污染的加剧，对环 境变化极为敏感的一些附生植物群落将因不适应新 的环境而逐渐消失。Gombert 等 (2004) 在法国南部 Grenoble 地区根据记录对酸和氮敏感的附生类地衣 分布情况来指示当地的空气纯度情况。当然, 附生 植物不仅仅是直接检验生态系统受污染破坏的生物
指示剂，还用来间接反映工业生产对人类健康的影 响(Szczepaniak \& Biziuk，2003)。在意大利 Loppi 等 (1998)通过 1980 与 1996 年两个时间段内对同一地 区的附生地衣的多度、多样性与微量元素的变化来 反映当地环境随着时间的变化。Esseen 和 Renhorn (1998) 通过对实验数据的分析后得出结论 认为可 将附生地衣的最大悬垂长度作为简捷的指标以衡量 森林边缘效应对附生植物群落的影响。

在不同程度的人为干扰或环境变化下, 森林群 落中附生植物的种类组成、生物量及其分布情况发 生明显的变化。通过对原生及受人为干扰的森林群 落中附生苔藓植物多样性与分布的比较研究, Vellak 和 Paal (1999)发现在受到人为干扰的森林中一些附 生苔藓种类消失, 附生苔藓物种类数量、物种多样性 指数及其分布区也明显地比原生森林减少。Casta (1999)报道了巴西南部地区原始热带雨林附生苔藓 的物种丰富度明显高于次生热带雨林。Barthlott 等 (2001) 通过观测研究, 注意到随着干扰程度的加大, 林冠附生植物的物种数呈下降的趋势, 反映出森林 环境条件变化对附生植物的影响。Benzing (1998)认 为作为附生植物集中分布的林冠层对气候变化最为 敏感。由此表明附生植物对环境变化干扰比较敏 感, 能够较好地反映林地环境质量状况。因此, 在很 大程度上，附生植物多样性、生物量及其分布变化都 能够较好地反应森林环境质量状况。目前,一些欧 美国家在开展森林生态系统结构与功能变化的动态 监测研究中已把有关附生植物生态学效应的研究作 为其中重要的内容 (Freiberg \& Freiberg, 2000 ; Lowman ,2001)。

此外，林冠附生植物群落所形成的垫层组织能 够为鸟类和生活在树上的其它动物提供栖息的场所 和食物 (Nadkarni \& Matelson，1989)。由于雾水与养 分条件将严重地制约附生植物的存在，它们的存在 吸引着很多寻求潮湿与庇护的动物。正是附生植物 的这种调节水分与养分的能力使得它们吸引了众多 的无脊椎动物和一些脊椎动物 (Benzing, 1998 ; Nadkarni \& Matelson，1989），从而维系了种子植物、乌 类、脊椎动物和微生物的多样性。此外, 这些垫层组 织还为寄主树的根系提供延展的空间 (Nadkarni， 1981）。虽然新近裸露的宿主树表面能在几个星期 的时间内被先锋附生植物所占据, 然而大多数能够 长期生存的附生植物却是生长在由苔藓和地衣所提 供的生长基质上 (Ibisch ,1996)。此外, 存在于树干、 石块的森林附生植物密布在其表面, 不仅前者能够 
对湿沉降中的养分进行选择性的截获，影响森林生 态系统的养分循环 (Liu et al. , 2002)，后者还减轻 了水土流失的程度。Nadkarni 和 Solano (2002) 试验 证明: 附生植物的死亡能导致林冠物种组成的改变, 因为附生植物死后留下的垫层上长满了陆生非附生 植物。说明附生植物所形成的垫状有机组织, 蕴涵 着丰富的种子, 是潜在的植物种子库; 同时附生植物 的存在阻碍了陆生种子植物在树上的萌发。尽管这 一说法一定程度上与附生植物对森林生态系统多样 性有贡献的说法相矛盾, 然而却起到了阻止外来物 种在森林中入侵的作用，尤其是那些靠风媒传播的 入侵物种。

5 影响林冠附生物组成、生物量与分布的主 要环境因子

附生植物的生长、发育、生殖和分布明显地受到 环境因子的影响。研究表明，环境因子作用于附生 植物群落将产生 3 种结果: 改变其分布, 改变其繁殖 力, 使其生长周期发生变化 (Laube \& Zotz, 2003)。 根据已有的研究表明, 影响林冠附生植物生长与分 布的环境因子主要是气候、地形因子和生长微环境 3 个方面。

\section{1 气象因子}

影响附生植物组成和分布的主要气候因子是水 分条件。尽管附生植物依靠特殊的结构在其组织中 咜备相当的水分以保证在干旱的条件下光合作用的 正常进行 (Benzing，1998)，但仍有一些学者认为水 分条件是制约附生植物分布最主要的因素,他们认 为附生植物适合于在降雨量大、空气湿度高、云雾天 气频繁和直射光弱的环境中生长 (Gentry \& Dodson , 1987 ; Hölscher et al. , 2004)。Nadkarni 和 Solaro (2002) 通过将哥斯达黎加山地云雾林中的附生植物 移栽到强光照、气候干燥的峡谷生境条件下, 模拟全 球气候变化对附生植物生长的影响, 试验结果表明 随着附生植物死亡率的增加，其生长速率明显降低， 表明水分条件对附生植物生长的影响显著。Leimbeck 和 Balslev (2001) 认为在湿度上即使是微小的变 化也会影响到附生植物群落的区系组成。Kreft 等 (2004) 也认为水分的作用严重影响着附生植物的生 存与繁衍; 水分因子比养分因子更能够制约附生植 物的生长, 这种制约的程度在附生植物处于幼年期 时显得格外明显(Zotz \& Hietz , 2001)。

大气降水对附生植物群落分布的影响作用相当 明显。以苔藓为代表的非维管束植物能否在各大森
林生态系统中建群分布主要是受降水的制约 (Benzing , 1998)。有学者证明降水的季节分配均匀与否 决定着附生植物分布的丰富程度, 例如在亚马逊的 热带雨林中，年降水量虽大于 $2000 \mathrm{~mm}$, 但是其附生 植物的生物量较安第斯山脉的大多数地区都少, 原 因在于前者每年都要经历一段干旱期 (Benzing, 1998)。最近的研究证实维管束附生植物的相对生 长量与年总降水量相关而不是与降水的天数相关 (Laube \& Zotz , 2003)。

此外, 通过野外调查和分析,Wolf (2003) 认为, 林冠上存在的风媒附生植物的相对高分布量与旱季 有关, 因为旱季有利于其种子的散布。这样的假说 说明风媒附生植物的存在很可能是干旱气候存在的 指示植物。

\section{2 地形因子}

有关地形因子对附生植物影响的研究主要是海 拔高度的变化。沿着海拔梯度的变化, 降水量、大气 湿度、大气温度、光照强度、风速和土壤条件等气象 生态因子呈现出较大的差异。Wolf (1993b) 研究了 安第斯山脉北侧森林附生物沿海拔梯度变化的情 况 发现附生苔藓植物在 2 550 3 $190 \mathrm{~m}$ 之间表现 出较高的物种多样性; 地衣的物种多样性在 1500 ３200 $\mathrm{m}$ 之间缓慢下降, 而在 $3200 \mathrm{~m}$ 以上又急剧 上升 林冠附生物生物量在 $2400 \mathrm{~m}$ 时显著增加, 与 湿度的迅速增大相一致，但与当地降水、散射光照变 化相关性低, 他认为林冠附生植物的组成与分布在 海拔梯度上的变化并不能用简单的函数关系来表 达，因为还受到其它诸多因素的制约。Freiberg 和 Freiberg (2000) 以单株树木为采样单位, 发现山地云 雾森林的附生植物物种数 ( $33 \sim 54$ 种/棵) 比低地森 林 (19 32 种/棵) 的多, 呈现出附生植物多度与丰 富度都随着海拔上升而增加的趋势。当然这种趋势 也不是绝对的, Gentry 和 Dodson (1987) 证实在高海 拔地区，降水量与附生植物物种丰富度并不存在正 相关关系。

\section{3 微生境}

微生境条件对林冠附生植物的生长和分布的影 响已逐渐为人们所重视。除了气候因素以外, 容纳 附生植物生长的微环境的类型和空间大小也影响着 附生植物的分布和丰富程度 (Esseen \& Renhorn， 1996)。Nieder 等 (2001) 认为, 影响附生植物在林冠 层中的分布的最主要因素是以枝条直径大小为形式 的生长基质条件。Ibisch (1996) 在玻利维亚山地雨 林中发现宿主树上由于枝条年龄的差异所导致生长 
基质的不同直接影响着附生植物的分布，在 10 年生 的枝条上能发现生活了 $6 \sim 8$ 年的凤梨科植物，在 10 $\sim 15$ 年的枝条上可以明显发现苔藓类的植物已经 将地衣类的植物排斥开了。在 $20 \sim 25$ 年的枝条上， 特别是当大的凤梨科植物死亡后留下了空隙可供所 有的新的附生植物建群生长。树高大小也是影响附 生植物多度的重要因素 (Lyons et al. , 2000)。此 外, 最新的研究表明, 树皮的 $\mathrm{pH}$ 值也是影响附生植 物群落的重要因素之一, 因为酸碱度不同的树皮上 出现了不同的附生植物种类 (Mitchell et al. , 2005)。在同一棵树上因不同部位微生境条件的不 同,所附生的植物种类和数量也分布不均匀。 Hölscher 等 (2004) 发现在林冠内部的生物量是在树 干上分布的 33 倍之多。Vellak 和 Paal (1999) 对爱 沙尼亚 Jarvselja 森林保护区中不同生长基质上的附 生苔藓植物进行的调查结果表明, 在活立木树干或 树基部、枯腐木和林地面上苔藓植物分别为 $19.9 \%$ 、 $14.9 \%$ 和 $65.2 \%$ 。

通过研究森林边缘效应对附生地衣生长与分布 的影响, 发现林缘与森林中心间在风速、光照、湿度 和大气养分粒子含量所存在的差异使得附生地衣 (Alectoria sarmentosa) 的多度存在着显著的差异 (Esseen \& Renhorn , 1998)。林窗动态演替过程中的微 环境的变化也会对森林附生植物带来影响, Padmawathe 等 (2004) 比较了不同的生境下附生维管束 植物在宿主树木上的盖度:末干扰密林中为 $82 \%$, 林窗为 $75 \%$,而伐木干扰区则为 $69 \%$ 。

\section{6 人为干扰对附生植物生长和分布的影响}

附生植物对人为活动的干扰很敏感。由于附生 植物生长速率低, 建群过程缓慢 (Laube \& Zotz, 2003) 对干扰的耐受范围相当有限, 所以轻微的干 扰便可影响到它们的生长与演替 (Nadkarni，2001； Nadkarni \& Solaro，2002)。森林附生植物群落一旦 遭受人为干扰破坏, 其恢复的过程将变得异常困难, 例如很多苔藓类和地衣类的附生植物因受到其繁殖 体传播能力较弱的限制，一旦生境经受任何干扰便 会使其在新的宿主树上建群的能力下降 (Vanderpoorten et al . 2004)。近年来人们对大自然无节制 的开发，使越来越多的原生植被遭到破坏，而呈现出 次生植被的面积越来越大的趋势。植被覆盖率的下 降, 伴随着光照加强、水平降水量减少、空气湿度降 低, 使得附生植物生长繁衍所需的特殊微环境减少， 继而导致其群落多度与多样性的减少。
在森林群落中, 有相当一部分树木不能为附生 植物提供适合的生长环境，尤其是那些先锋树种，所 以森林在受到干扰后, 先锋树种的出现会大量地削 减附生植物的生物量 (Romero，1999)。Barthlott 等 (2001) 注意到随着人为干扰程度的增大，林冠附生 植物的物种数量有下降的趋势。有报道显示新加坡 有 $62 \%$ 的附生植物物种经过大强度的毁林后绝种 了(Turner et al. , 1994)。Pykala(2003) 发现在芬兰 南部 Lohja 地区森林中由于人为开圼耕地所造成的 森林生境片断化(耕地占 $22.5 \%$ ) 使得当地地衣种 群森林破坏后的 10 年内因宿主树木的砍伐而消失 了近 $20 \%$ 。

由于长期以来人为干扰和破坏, 很多原始森林 面积减少, 许多已演变成次生林、灌丛或荒草地, 生 境条件发生明显变化。Barthlott 等 (2001) 认为在热 带地区即便是面积很小的原生林植被都将起到重要 的维系附生植物多样性的作用。在经过伐木干扰的 森林中, 温度与光照强度均比在原生林和其中的林 窗中大, 而相对湿度却明显降低 (Padmawathe et al. , 2004)。受严重干扰的次生林，林木稀疏，林冠层中 光照强、空气湿度小, 从而降低了耐荫附生植物的多 度、分布和多样性 (Hietz-Seiferet et al , , 1996)。此 外，在次生林中大径级宿主树木少，不能为附生植物 的建群提供足够的生长微环境，也是造成附生植物 的分布和组成在两种生境下差异悬殊的原因。当 然，仅仅对附生植物彼此间的多度、盖度、丰富度和 生物量在数值上作比较, 而不结合它们各自在系统 中的地位和作用是不足以说明附生植物群落在不同 生境下的差异的。因为 Nadkarni 等 (2004) 发现在原 生林中的附生物质能够截获大气中的养分元素、为 林冠无脊椎动物提供栖息地以及为动物提供食物来 源等,而在次生林中却不具备这样的功能。

人们对森林的不同经营管理手段也会影响到附 生植物群落组成和分布的改变。Vellak 和 Paal (1999)的调查结果表明，在未经管理的保护区森林 和实施管理的人工林中分别有 74 和 54 种苔藓植物 出现, 虽然在苔藓植物分布多度和生长基质 (Substrate) 类型等指标上均不存在统计意义上的显著差 异, 但作者认为具有附生特性的苔藓植物在未经管 理的天然林比有人工管理的森林中数量要多, 说明 人为经营管理措施对森林中附生植物群落结构产生 较大的影响。

\section{7 林冠附生植物研究展望}

基于对林冠附生植物多方面的研究结果, 许多 
学者认为林冠附生植物及其枯死残留物在维系生态 系统生物多样性格局，以及养分、水分循环方面起到 了重要的作用, 应对生境潮湿的热带和温带山地森 林生态系统的结构和功能进行重新评价 (Knops et al . , 1991 ; Lowman \& Nadkarni , 1995 ; Ozanne et al. , 2003 ; Hölschera et al . 2004)。另一方面, 在国际上 新兴的 林冠学” 研究和现代 生态林业”理论及管理 中, 也把了解林冠附生生物组成、结构和生物量及其 生态学效应作为山地森林资源动态和环境研究及管 理的重要基础之一 ( Gillis, 1990 ; Lowman \& Nadkarni , 1995 ; Lowman , 2001)。

虽然林冠附生植物生态学研究取得了很大的进 展 然而,由于世界各地森林类型和环境条件等方面 的差异，加之人们对森林附生植物的了解远远少于 对其它植物的了解，目前，国际上对林冠附生物的研 究仍十分活跃。一方面, 他们研究不同类型森林中 附生物结构与功能的动态, 其中对不同类型森林附 生植物的多样性格局及其形成机制，活、死附生植物 的生物量及其空间分布特性, 附生植物与不同宿主 之间、生态因子之间的关系、附生植物获取大气营养 元素(包括某些物种自身具备固氮功能)的能力, 对 环境变化或人为活动干扰的忍受能力等等, 仍然是 目前森林附生物研究中的热点问题 (Lowman \& Ndkarni ,1995; Lowman，2001)。另一方面，因为森林附 生物主要分布于林冠层中, 故探讨一种林冠附生物 质的标准测量方法, 发展利用统计学的方法和技术, 研究从树木个体到生态系统和景观空间尺度上附生 植物组成、生物量分布变化规律, 也成为现阶段森林 附生植物研究的重要内容之一（Lowman \& Ndkarni, 1995)。

\section{8 我国有关林冠附生植物研究的现状和建议}

在我国, 过去对林冠附生植物的研究主要集中 在附生苔藓、偋类、地衣和种子植物的物种鉴定和有 关附生植物区系地理分布方面，为探讨和揭示中国 植物区系的形成、发生和发展起了十分积极的作用。 然而，对林冠附生植物及其枯死残留物的生态学效 应了解不多。近年来，林冠附生物在森林生态系统 中的重要作用已开始逐渐引起有关学者注意。在注 重吸取前人研究成果的基础上,开展了有关附生植 物多样性、生物量、养分和水分含量的研究, 并将其 生态学意义的认识提高到对生态系统影响和作用的 层面上 (汪庆等, 1999;刘文耀,2000)。曹同等对长 白山森林中 $1.5 \mathrm{~m}$ 高度以下的附生苔藓植物的生物
量、多样性及其分布规律 (曹同和郭水良, 2000; 郭水 良和曹同, 2000a, 2000b) 进行了研究。刘文耀等对 山地湿性常绿阔叶林中的附生苔藓植物的分解速 率、生态功能进行了初步的研究 ( Liu et al. , 2000, 2002)。最近对云南哀牢山山地湿性常绿阔叶林林 冠附生植物群落的物种多样性与空间分布特性、附 生植物与宿主关系等进行了较为详细的研究 (徐海 清和刘文耀, 2005) , 为全面、深入地研究该地区山地 湿性森林生态系统结构与功能以及生物多样性形成 格局提供了科学依据。

然而，我国地域辽阔，多样化的气候和复杂的地 理环境, 孕育着丰富多样的森林类型。以往我国在 开展山地森林生态系统结构与功能研究中, 特别是 对热带、亚热带和温带高海拔地区山地湿性森林生 态系统生产力形成机制及其功能过程进行研究时, 没有把附生植物及其枯死残留物生物量纳入到森林 总生物量中去，对森林中附生物的组成、生物量空间 分布, 附生物与宿主和生境变化的关系, 以及附生物 在生态系统水分、养分循环中的作用及其影响机制 等还了解很少, 很显然, 这对了解山地森林生产力结 构、生物多样性格局及其维持机制，以及森林资源的 科学经营管理是不全面的, 缺乏深度。因此, 在全 面、深入、持续地研究热带、亚热带和温带山地森林 生态系统的结构、功能、动态特征及管理途径过程 中, 需重视林冠附生物物种多样性、生产力及其生态 功能的研究, 以了解它们在森林生态系统过程中的 作用及其机制。

\section{参 考 文 献}

Annasel VJ, Parthasarathy N (2001). Diversity and distribution of herbaceous vascular epiphytes in a tropical evergreen forest at Varagalaiar, Western Ghats, India. Biodiversity and Conservation, $10,317-329$.

Baker GM, Pinard AM (2001). Forest canopy research: sampling problems, and some solutions. Plant Ecology, 153, 23-38.

Barkman JJ (1958). Phytosociology and Ecology of Cryptogamic Epiphytes. Van Gorcum, Assen, Netherlands.

Barthlott W, Schmit-Neuerburg V, Nieder J, Engwald S (2001).

Diversity and abundance of vascular epiphytes: a comparison of secondary vegetation and primary montane rain forest in the Venezuelan Andes. Plant Ecology, 152, 145 - 156.

Basset Y, Hammond PM, Barrios H, Holloway JD, Miller SE

(2003a). Vertical stratification of arthropod assemblages. In: Basset Y, Kitching R, Miller S, Novotny V eds. Arthropods of Tropical Forests: Spatio-Temporal Dynamics and Resource Use in the Canopy. Cambridge University Press, Cambridge, 17-27.

Basset Y, Horlyck V, Wright SJ (2003b). Executive summary. 
In: Basset Y, Horlyck V, Wright SJ eds. Studying Forest Canopies from Above: the International Canopy Crane Network. Smithsonian Tropical Research Institute and UNEP, Panama, 21 $-23$.

Basset Y, Horlyck V, Wright SJ (2003c). The conservation of forest canopies: policy and science. In: Basset Y, Horlyck V, Wright SJ eds. Studying Forest Canopies from Above: the International Canopy Crane Network. Smithsonian Tropical Research Institute and UNEP, Panama, 37 - 54.

Benzing DH (1998). Vulnerabilities of tropical forest to climate change: the significance of resident epiphytes. Climatic Change, $39,519-540$.

Berg T, Steinnes E (1997). Recent trends in atmospheric deposition of trace elements in Norway as evident from the 1995 moss survey. Science of the Total Environment, 208, $197-206$.

Brown DH ( 1982 ). Mineral nutrition. In: Smith AJE ed. Bryophyte Ecology. Chapman and Hall, London, 383 - 444.

Cao T (曹同), Guo SL (郭水良) (2000). A study on bryophytes diversity in the main ecosystem in Changbai Mountain. Chinese Biodiversity (生物多样性), 8, 50-59. (in Chinese with English abstract)

Casta DP (1999) . Epiphytic bryophyte diversity in primary and secondary lowland rainforests in Southeastern Brazil. The Bryologist, $102,320-326$.

Coxson DS (1991). Nutrient release from epiphytic bryophyte in tropical montane rain forest (Guadeloupe) . Canadian Journal of Botany, 69, 2122 - 2129.

Coxson DS, Nadkarni NM (1995). Ecological roles of epiphytes in nutrient cycles of forest ecosystems. In: Lowman MD, Nadkarni NM eds. Forest Canopy. Academic Press, San Diego, 495 543.

Edward PJ, Grubb PJ (1977). Studies of mineral cycling in a montane rain forest in New Guinea. I. The distribution of organic matter in the vegetation and soil. Journal of Ecology, 65, $943-$ 967.

Esseen P, Renhorn K (1996). Epiphytic lichen biomass in managed and old growth boreal forests: effect of branch quality. Ecological Applications, 6, 228-238.

Esseen P, Renhorn K (1998). Edge effects on an epiphytic lichen in fragmented forests. Conservation Biology, 12, $1307-1317$.

Field TS, Dawson TE (1998). Water sources used by Didymopanax pittieri at different life stages in a tropical cloud forest. Ecology, 79, 1448 - 1452 .

Freiberg M, Freiberg E (2000). Epiphyte diversity and biomass in the canopy of lowland and montane forest in Ecuador. Journal of Tropical Ecology, 16,673-688.

Gentry AH, Dodson CH (1987). Diversity and biogeography of neotropical vascular epiphytes. Annals of the Missouri Botanical Garden, 74, 205 - 233.

Gillis AM (1990). The new forestry: an ecosystem approach to land management. Biotropica, 40, 558-562.
Gombert S, Asta J, Seaward MRD (2004). Assessment of lichen diversity by index of atmospheric purity (IAP), index of human impact (IHI) and other environmental factors in an urban area (Grenoble, southeast France). Science of the Total Environment, $324,183-199$.

Guo SL (享水良), Cao T (曹同) (2000a). Studies on community distributive patterns of epiphytic bryophytes in forest ecosystems in Changbai Mountain. Acta Phytoecologica Sinica (植物生态学 报), 24, 442-450. (in Chinese with English abstract)

Guo SL (郭水良), Cao T (曹同) (2000b). Studies on relationship of epiphytic bryophytes and environmental factors in forest ecosystems in Changbai Mountain forests. Acta Ecologica Sinica (生态学报), 20,924 - 931. (in Chinese with English abstract)

Hall JB (1978) . Checklist of the vascular plants of Bia National Park and Bia game production reserve. In: Martin C ed. Management Plan for the Bia Wildlife Conservation Areas. Part I. Wildlife and National Parks Division, Ghana Forestry Commission. Final Report IUCN/WWF Project, 1251.

Hammond PM, Stork NE, Brendell MJD (1997). Tree-crown beetles in context: a comparison of canopy and other ecotone assemblages in a lowland tropical forest in Sulawesi. In: Stork NE, Adis J, Didham R eds. Canopy Arthropods. Chapman \& Hall, London, $184-223$.

Hietz P, Wanek W, Popp M (1999). Stable isotopic composition of carbon and nitrogen, and nitrogen content in vascular epiphytes along an altitudinal transect. Plant, Cell and Environment, 22, $1435-1443$.

Hietz-Seiferet U, Heitz P, Guevara S (1996). Epiphyte vegetation and diversity on remnant trees after forest clearance in southern Veracruz, Mexico. Biological Conservation, 75, 103-111.

Höfstede RGM, Dickinson KJM, Mark AF (2001). Distribution, abundance and biomass of epiphyte-lianoid communities in a New Zealand lowland Nothofagus-Podocarp temperate rain forest: tropical comparisons. Journal of Biogeography, 28, 1033 - 1049 .

Höfstede RGM, Wolf J, Benzing DH (1993). Epiphytic biomass and nutrient status of a Colombian Upper Montane Rain Forest. Selbyana, 14, $37-45$.

Hölscher D, Köhlera L, van Dijk AIJM, Bruijnzeel LA (2004) . The importance of epiphytes to total rainfall interception by a tropical montane rain forest in Costa Rica. Journal of Hydrology, $292,308-322$.

Hopkin M (2005) . Biodiversity and climate form focus of forest canopy plan. Nature, 436, 452 .

Ibisch PL (1996). Neotropical Epiphyte Diversity-the Bolivian Example. Ph D dissertation, University of Bonn, Bonn, Alemania, Martina Galunder-Verlag, 1 - 470.

Jarvis A (2000) . Measuring and modeling the impact of land-use change in tropical hillsides: the role of cloud interception to epiphytes. Advances in Environmental Monitoring and Modeling, 1, $118-148$. 
Jenik J (1973) . Root systems of tropical trees. 8. Stilt-roots and allied adaptations. Preslia, 45, 250-264.

Knops JMH, Nash TH, Boucher VL, Schlesinger WH ( 1991 ). Mineral cycling and epiphytic lichens: implication at ecosystem level. Lichenologist, 23, 309-321 .

Kreft H, Köster N, Küper W, Nieder J, Barthlott W (2004) . Diversity and biogeography of vascular epiphytes in Western Amazonia, Yasuní, Ecuador. Journal of Biogeography, 31, 1463 1476.

Kress WJ (1986). The systematic distribution of vascular epiphytes: an update. Selbyana, 9, 2-22.

Laube S, Zotz G (2003). Which abiotic factors limit vegetative growth in a vascular epiphyte? Functional Ecology, 17, 598 604.

Leimbeck RM, Balslev H (2001). Species richness and abundance of epiphytic Araceae on adjacent floodplain and upland forest in Amazonian Ecuador. Biodiversity and Conservation, 10, 1579 1593.

Liu WY (刘文耀) (2000). The role of epiphytic material in nutrient cycling of forest ecosystem. Chinese Journal of Ecology (生 态学杂志), 19, 30-35. (in Chinese with English abstract)

Liu WY, Fox JED, Xu ZF (2000). Leaf litter decomposition of canopy trees, bamboo and moss in a montane moist evergreen broad-leaved forest on Ailao Moutian, Yunnan, South-West China. Ecological Research, 15, 435 - 447.

Liu WY, Fox JED, Xu ZF (2002) . Nutrient fluxes in bulk precipitation, throughfall and stemflow in montane subtropical moist forest on Ailao Moutians in Yunnan, SW China. Journal of Tropical Ecology, 18, 527 - 548 .

Loppi S, Putorti E, Signorini C, Fommei S, Pirintsos SA, de Dominicis V (1998). A retrospective study using epiphytic lichens as biomonitors of air quality: 1980 and 1996 (Tuscany, central Italy). Acta Oecologia, 19, $405-408$.

Lowman MD, Nadkarni NM (1995). Forest Canopy. Academic Press, San Diego.

Lowman MD (2001). Plant in the forest canopy: some reflections on current research and future direction. Plant Ecology, 153, 39 -50 .

Lugo AE, Scatena F (1992). Epiphytes and climate change research in the Caribbean: a proposal. Selbyana, 13, 123-130.

Lyons B, Nadkarni MN, North PM (2000). Spatial distribution and succession of epiphytes on Tsuga heterophylla (western hemlock) in an old-growth Douglas-fir forest. Canadian Journal of Botanty, 78, $957-968$.

Mitchell RJ, Truscot AM, Leith ID, Cape JN, van Dijk N, Tang YS, Fowler D, Sutton MA (2005). A study of the epiphytic communities of Atlantic oak woods along an atmospheric nitrogen deposition gradient. Journal of Ecology, 10, 1 - 10 .

Nadkarni NM (1981). Canopy roots: convergent evolution in rainforest nutrient cycles. Science, 214, $1023-1024$.

Nadkarni NM (1984). Epiphyte biomass and nutrient capital of a neotropical elfin forest. Biotropica, 16, $249-256$.

Nadkarni NM (1985). Biomass and nutrient capital of epiphyte in an Acer macrophyllum community of a temperate moist coniferous forest, Olympic Peninsula, Washington State. Canadian Journal of Botany, 62, 2223 - 2228 .

Nadkarni NM, Matelson TJ (1989) . Bird use of epiphyte resources in Neotropical trees. The Condor, 91, $891-907$.

Nadkarni NM, Matelson TJ (1992). Biomass and nutrient dynamics of epiphyte litterfall in a neotropical cloud forest, Costa Rica. Biotropica, 24, $24-30$.

Nadkarni NM (2001). Forest canopies plant diversity. Encyclopedia of Biodiversity, 3, $27-40$.

Nadkarni NM, Solano R (2002) . Potential effects of climate change on canopy communities in a tropical cloud forest: an experimental approach. Oecologia, 131, 580-586.

Nadkarni NM, Schaefer DA, Matelson TJ, Solano R (2004). Biomass and nutrient pools of canopy and terrestrial components in a primary and a secondary montane cloud forest, Costa Rica. Forest Ecology and Management, 198, 223 - 236.

Nieder J, Zotz G (1998). Methods of analyzing the structure and dynamics of vascular epiphyte communities. Ecotropica, 4, 33 39.

Nieder J, Prosper J, Michaloud G (2001). Epiphytes and their contribution to canopy diversity. Plant Ecology, 153, $51-63$.

Ozanne CMP, Anhuf D, Boulter SL, Keller M, Kitching RL, Körner C, Meinzer FC, Mitchell AW, Nakashiyuka T, Silva Dias PL, Stork NE, Wright SJ, Yoshimura M (2003). Biodiversity meets the atmosphere: a global view of forest canopies. Science, $301,183-186$.

Økland RH (1994). Patterns of bryophyte associations at different scales in a Norwegian boreal spruce forest. Journal of Vegetation Science, 5, $127-138$.

Padmawathe R, Qureshi Q, Rawat GS (2004). Effects of selective logging on vascular epiphyte diversity in a moist lowland forest of Eastern Himalaya India. Biological Conservation, 119, 81 - 92.

Parker GG (1995). Structure and microclimate of forest canopies. In: Lowman MD, Nadkarni NM eds. Forest Canopy. Academic Press, San Diego, 73 - 106.

Peck JE, McCune B (1998). Commercial moss harvest in northwestern Oregon: biomass and accumulation of epiphytes. Biological Conservation, 86, $299-305$.

Pennisi E (2005). Sky-high experiments. Science, 309, 1314 1315 .

Pentecost A (1998) . Some observation on the biomass and distribution of cryptogamic epiphytes in the upper montane forest of the Rwenzori Mountains, Uganda. Global Ecology and Biogeography Letters, 7, $273-284$.

Perry DR (1978). A method of access into the crowns of emergent and canopy trees. Biotropica, 10, $155-157$.

Pócs T (1980). The epiphytic biomass and its effect on the water balance of two rainforest types in the Ulugruru Mountains. Acta 
Botanica Academiae Scientiarum Hungaricae, 26, 143 - 167.

Pykala J (2003) . Effects of new forestry practices on rare epiphytic macrolichens. Conservation Biology, 18, $831-838$.

Rodgers DJ, Kitching RL (1998). Vertical stratification of rainforest collembolan (Collembola: Insecta) assemblages: description of ecological patterns and hypotheses concerning their generation. Ecography, 21, 392 - 400 .

Romero C (1999) . Reduced-impact logging effects on commercial non-vascular pendant epiphyte biomass in a tropical montane forest in Costa Rica. Forest Ecology and Management, 118, 117 125 .

Silvola J, Aaltonen H (1984). Water content and photosynthesis in peat mosses Sphagnum fuscum and $S$. angustifolium. Annual Botany of Fennic, 21, $1-6$.

Sloof JE (1993) . Environmental Lichenology: Biomonitoring TraceElement Air Pollution. Ph D dissertation, Delft University of Technology, Netherland.

Songwe NC, Fasehun FE, Okali DU (1998). Litterfall and productivity in a tropical rain forest, Southern Bakundu Forest Reserve, Cameroon. Journal of Tropical Ecology, 4, 25-37.

Sorensen LL (2003). Stratification of spider fauna in a Tasmanian forest. In: Basset Y, Kitching R, Miller S, Novotny V eds. Arthropods of Tropical Forests: Spatio-temporal Dynamics and Resource Use in the Canopy. Cambridge University Press, Cambridge, $92-101$.

Stuntz S, Simon U, Zotz G (2002). Rainforest air-conditioning: the moderating influence of epiphytes on the microclimate in tropical tree crowns. International Journal of Biometeorology, 46, 53 -59 .

Szczepaniak K, Biziuk M (2003). Aspects of the biomonitoring studies using mosses and lichens as indicators of metal pollution. Environmental Research, 93, 221 - 230 .

Tanner EVJ (1980). Studies on the biomass and productivity in a series of montane rain forests in Jamaica. Journal of Ecology, $68,573-588$.

Turner IM, Tan HTW, Wee YC, Ibrahim AB, Chew PT, Corlett RT (1994). A study of plant species extinction in Singapore: Lessons for the conservation of tropical biodiversity. Conservation Biology, 8, $705-712$.
Vanderpoorten A, Engels P, Sotiaux A (2004). Trends in diversity and abundance of obligate epiphytic bryophytes in a highly managed landscape. Ecography, 27, 567 - 576.

Vellak K, Paal J (1999). Diversity of bryophyte vegetation in some forest types in Estonia: a comparison of old unmanaged and managed forests. Biodiversity and Conservation, 8, 1595 - 1620 .

Veneklaas E (1991). Litterfall and nutrient fluxes in two montane tropical rain forests, Colombia. Journal of Tropical Ecology, 7, $319-335$.

Vitousek PM, Sanford RL Jr (1986). Nutrient cycling in moist tropical forest. Annual Review of Ecology and Systematics, 17, $137-167$.

Wang Q (汪庆), He SA (贺善安), Wu PC (吴鹏程) (1999). The role bryophytes in biodiversity. Chinese Biodiversity (生物多 样性) , 7, 332 - 339. (in Chinese with English abstract)

Weathers KC (1999). The importance of cloud and fog in the maintenance of ecosystems. Trends in Ecology \& Evolution, 14, 214 -215 .

Wolf JHD (1993a). Ecology of Epiphytes and Epiphyte Communities in Montane Rain Forest, Colombia. Ph D dissertation, University of Amsterdam, Netherlands.

Wolf JHD (1993b). Diversity patterns and biomass of epiphytic bryophytes and lichens along an altitudinal gradient in the northern Andes. Annual Missouri Botanic Gardens, 84, 928 - 960 .

Wolf JHD (2003). Patterns in species richness and distribution of vascular epiphytes in Chiapas, Mexico. Journal of Biogeography, 30, $1689-1707$.

Wolf JHD (2005). The response of epiphytes to anthropogenic distribution of pine-oak forests in the highlands of Chiapas, Mexico. Forest Ecology and Management, 212, 376 - 393.

Xu HQ (徐海清), Liu WY (刘文耀) (2005). Species diversity and distribution of epiphytes in the montane moist evergreen broad-leaved forest in Ailao Mountain, Yunnan. Biodiversity Science (生物多样性), 13, 137 - 147. (in Chinese with English abstract)

Zotz G, Hietz P (2001). The ecophysiology of vascular epiphytes: current knowledge, open questions. Journal of Experimental Botany, 52, $2067-2078$. 